CASO CLÍNICO

\title{
Síndrome de Bland-White-Garland en una mujer adulta mayor. Reporte de un caso
}

\section{Bland-White-Garland syndrome in a senior adult woman. Case report}

Sonia L. Gómez-Briones ${ }^{1 *}$, Agustín Fraga-Ramos ${ }^{1}$, Martha Morelos-Guzmán ${ }^{1}$ y Edgar Paulo-Valdivieso²

${ }^{1}$ Servicio de Cardioimagen; ${ }^{2}$ Servicio de Imagen. Hospital General Dr. Miguel Silva, Michoacán, México

\section{RESUMEN}

El origen anómalo de la arteria coronaria izquierda a partir de la arteria pulmonar, también conocido como síndrome de Bland-White-Garland, es una cardiopatía congénita infrecuente. Se asocia con mortalidad infantil temprana, y en adultos, con muerte súbita. Su incidencia se estima entre el 0.2 y el $0.4 \%$ en nacidos vivos y en $0.008 \%$ en adultos. Presentamos el caso de una mujer de 66 años con antecedentes de hipertensión y dislipidemia, que clínicamente presentó dolor precordial. En la angiotomografía se observa el origen anómalo de la arteria coronaria izquierda de la arteria pulmonar, además de circulación colateral intercoronaria ectásica.

Palabras clave: ALCAPA. Síndrome de Bland-White-Garland. Congénito. Coronarias.

\section{ABSTRACT}

The anomalous origin of the left coronary artery from the pulmonary artery (ALCAPA) also known as Bland-White-Garland syndrome, is a rare congenital heart disease, associated with early infant mortality and sudden death in adults. Its incidence is estimated between 0.2 to $0.4 \%$ in live births and $0.008 \%$ in adults. We present the case of a 66 -yearold woman with a history of hypertension and dyslipidemia, who presented with precordial pain. In the angiotomography, the anomalous origin of the left coronary artery of the pulmonary artery is observed, in addition to ectasic intercoronary collateral circulation.

Key words: ALCAPA. Bland-White-Garland syndrome. Congenital. Coronary. 


\section{INTRODUCCIÓN}

El origen anómalo de la arteria coronaria izquierda a partir de la arteria pulmonar (ALCAPA), también conocido como síndrome de Bland-White-Garland, es una cardiopatía congénita infrecuente asociada con mortalidad infantil temprana, y en la edad adulta, con muerte súbita ${ }^{1}$.

La incidencia de ALCAPA se estima en $1 / 300,000$ nacidos vivos, que comprende entre el $0.24 \%$ y el $0.46 \%$ de la enfermedad cardíaca congénita $^{2,3}$.

Regularmente se presenta como un defecto aislado, pero en el 5\% de los casos puede estar asociado con otras anomalías cardíacas como la comunicación interauricular, la comunicación interventricular y la coartación aórtica ${ }^{4}$.

Su mortalidad es elevada, aproximadamente el $90 \%$ de los casos sin tratamiento fallecen durante el primer año de vida, y el 10\% restante pueden alcanzar la edad adulta ${ }^{5}$.

\section{CASO CLÍNICO}

Mujer de 66 años, ama de casa, con antecedentes de hipertensión arterial, dislipidemia en tratamiento y neumopatía por exposición al humo de leña. No refiere antecedentes patológicos familiares.

Consulta por dolor precordial atípico de 6 meses de evolución relacionado con el esfuerzo físico moderado, que se acompaña de palpitaciones ocasionales. Al examen físico durante la auscultación se destaca la presencia de soplos de insuficiencia aortomitral.

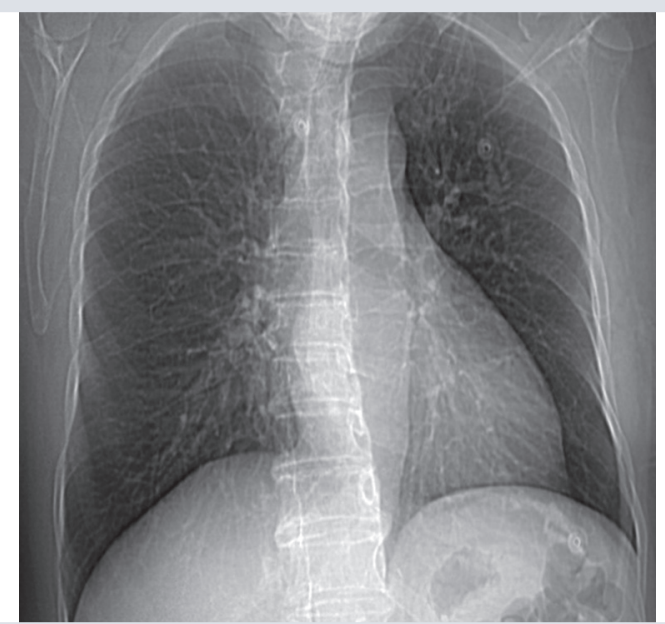

Figura 1. Radiografía de tórax proyección PA. Se evidencia cardiomegalia grado I- II a expensas de cavidades izquierdas, ensanchamiento del mediastino, cambios de angioesclerosis del botón aórtico y sobredistensión pulmonar. PA: posteroanterior.

La radiografía de tórax muestra cardiomegalia, cambios de angioesclerosis del botón aórtico y sobredistensión pulmonar (Fig. 1).

El electrocardiograma muestra ritmo sinusal, onda $\mathrm{T}$ negativa de ramas simétricas de $\mathrm{V} 1 \mathrm{a}$ V4, DI y aVL sugestivo de isquemia miocárdica (Fig. 2).

El ecocardiograma transtorácico reportó situs solitus, concordancia auriculoventricular y ventriculoarterial, con un ventrículo izquierdo dilatado, y destaca hipertrofia excéntrica del mismo. No se apreciaron alteraciones de la contractilidad segmentaria. Función sistólica global conservada. Fracción de eyección ventricular izquierda del 53\%. Dilatación de ambas aurículas e insuficiencia valvular aortomitral leve.

La angiotomografía cardíaca mostró el origen anómalo del tronco coronario izquierdo 


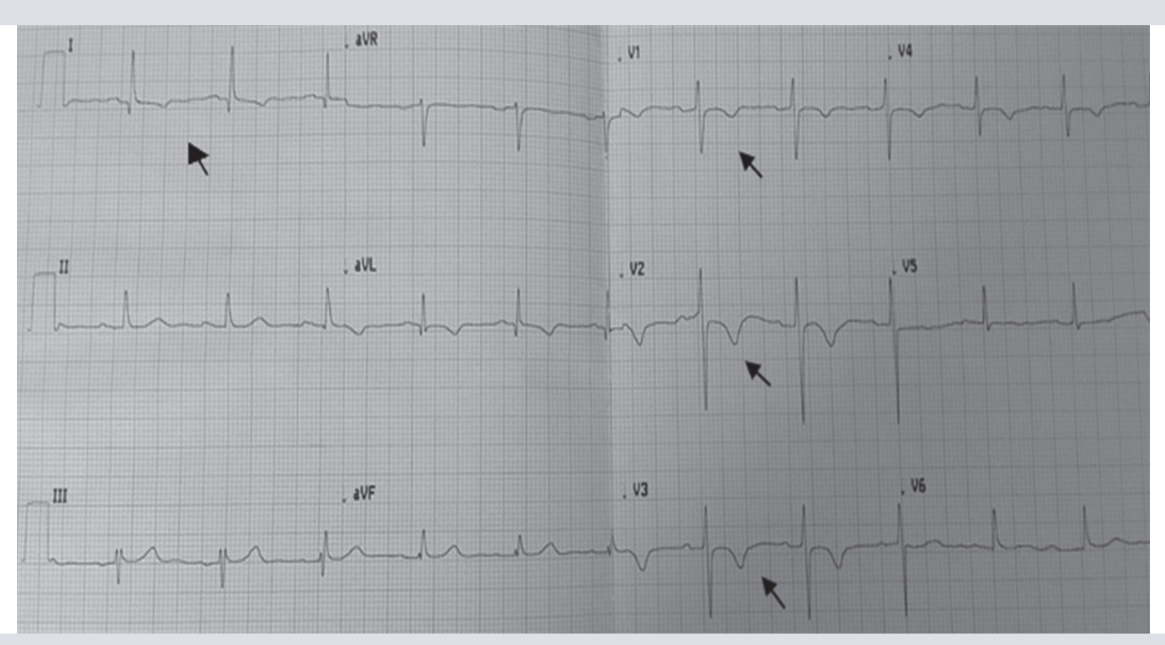

Figura 2. Electrocardiograma realizado en el servicio de urgencias. El electrocardiograma de 12 derivaciones muestra ritmo sinusal con FC de $70 \mathrm{lpm}$, onda T negativa de ramas simétricas de V1 a V4, DI y aVL sugestivo de isquemia miocárdica. FC: frecuencia cardíaca.

(*) a partir de la arteria pulmonar (AP) (ALCAPA) (Fig. 3). El ventrículo izquierdo se encuentra dilatado sin alteraciones de la movilidad y función sistólica global conservada (Fig. 4). La arteria coronaria derecha (CD) de origen normal, trayecto tortuoso y ectásico, con un diámetro transverso de $12 \mathrm{~mm}$. Presenta placas calcificadas excéntricas, no significativas. Además se muestra una red de circulación colateral intercoronaria dilatada a consecuencia de la inversión del flujo (Fig. 5).

\section{DISCUSIÓN}

El ALCAPA también se conoce como síndrome de Bland-White-Garland debido a que los médicos William Franklin Bland, Paul Dudley White y Joseph Garland del Hospital General de Massachusetts (Boston), en 1933, fueron quienes realizaron la primera descripción clínica ${ }^{1}$.
El ALCAPA es una anomalía del origen del ostium del tronco coronario izquierdo, el cual se presenta predominantemente en el primer año de vida, con una incidencia aproximada de 1/300,000 nacidos vivos, y representa entre el 0.26 y $0.46 \%$ de las cardiopatías congénitas. En adultos vivos es extremadamente raro, con una incidencia estimada del $0.008 \%{ }^{2,3}$.

Durante la vida fetal y neonatal, el ALCAPA puede ser bien tolerado, debido a que la presión tanto pulmonar como sistémica son iguales, por lo que el flujo es anterógrado. Tras el nacimiento, cuando la presión en la arteria pulmonar disminuye, el flujo a través de la arteria coronaria izquierda es menor y finalmente acaba invirtiéndose («robo coronario»), y genera hipoperfusión e isquemia miocárdica ${ }^{4}$. Debido a esto, el $90 \%$ de los afectados fallecen en el primer año de vida.

Sin corrección quirúrgica, menos del 10\% alcanzan la edad adulta, y los escasos 

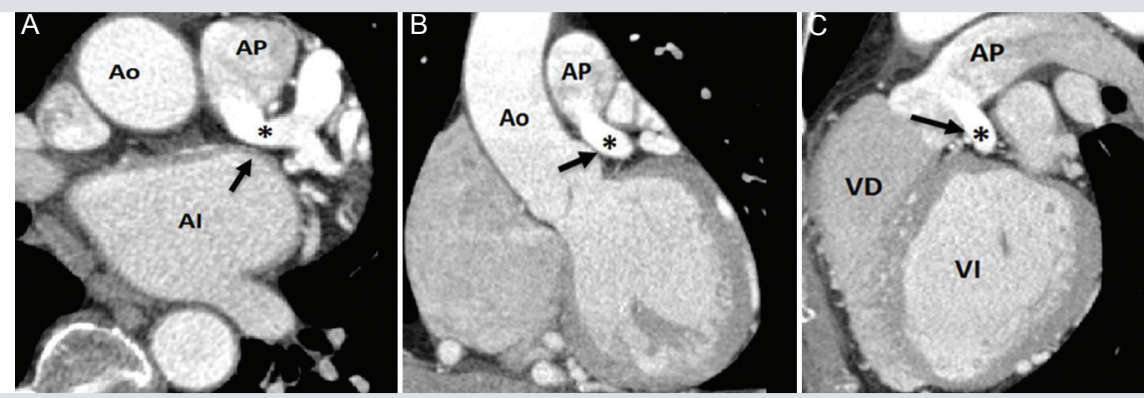

Figura 3. Síndrome del origen anómalo de la arteria coronaria izquierda a partir de la arteria pulmonar en una mujer de 66 años. La angiotomografía en una vista axial. A: oblicua de tres cámaras en eje largo. B: curva multiplanar. C: muestra el origen anómalo del tronco coronario izquierdo $\left({ }^{*}\right)$ a partir de la arteria pulmonar (AP).
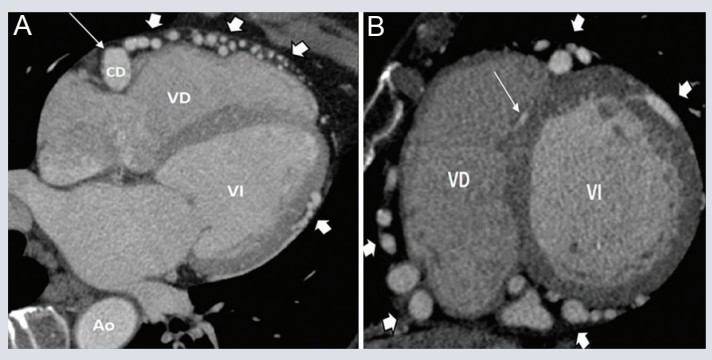

Figura 4. Angiotomografía en una vista axial de cuatro cámaras. A: oblicua en eje corto de dos cámaras. B: muestra los vasos colaterales intercoronarios dilatados a lo largo de la superficie epicárdica (flechas cortas), además de la hipertrofia ventricular izquierda sin trombos intracavitarios en su interior.

pacientes que sobreviven lo hacen gracias a la formación de numerosas colaterales entre las arterias coronarias derecha e izquierda que aportan una irrigación suplementaria al miocardio ${ }^{5}$.

Su forma de presentación clínica es variada, y depende del desarrollo de la circulación colateral que se establezca. El 80 a $85 \%$ de los pacientes sin un suministro colateral adecuado generalmente presentan insuficiencia cardíaca congestiva, secundaria a la isquemia en

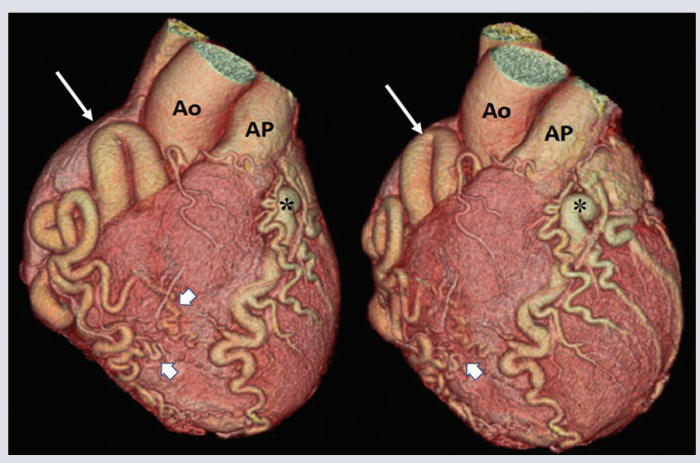

Figura 5. Angiotomografía cardíaca 3D que muestra la circulación colateral intercoronaria ectásica (flechas cortas) que conecta la arteria coronaria derecha tortuosa y ectásica (flecha larga) con la coronaria izquierda $\left({ }^{*}\right)$, la cual se origina a partir de la arteria pulmonar.

los primeros meses de vida. Su tasa de mortalidad es del 90\% sin corrección quirúrgica temprana ${ }^{3}$, mientras que los pacientes con un suministro colateral adecuado pueden permanecer asintomáticos hasta la edad adulta y luego presentar un soplo (generalmente continuo) en el borde paraesternal izquierdo, disfunción ventricular izquierda, regurgitación mitral, angina de esfuerzo, y están en riesgo de muerte cardíaca súbita ${ }^{1}$. 
Tradicionalmente, el ALCAPA ha sido diagnosticado por angiografía o autopsia; sin embargo, el desarrollo de la tomografía computarizada y la resonancia magnética cardíacas han permitido una evaluación no invasiva de la anatomía coronaria.

Los hallazgos de TC y RM cardíacas de ALCAPA en adultos incluyen la visualización directa del origen de la arteria coronaria izquierda desde la cara posterior de la arteria pulmonar, la arteria coronaria derecha dilatada, tortuosa, y la visualización de colaterales intercoronarias dilatadas a lo largo de la superficie externa del corazón o dentro del tabique interventricular, como es el caso de nuestra paciente ${ }^{6,7}$.

Ambas modalidades pueden emplearse para evaluar la función sistólica del ventrículo izquierdo. La RM tiene la ventaja adicional de demostrar la comunicación y el flujo desde la arteria coronaria izquierda hacia la arteria pulmonar. Es útil para evaluar la función valvular mitral, y el realce tardío con gadolinio puede ayudar a determinar la viabilidad miocárdica ${ }^{8}$.

Ambas técnicas de imagen son útiles, no solo en el diagnóstico, sino que también ofrecen información pronóstica, permiten la estratificación del riesgo y se pueden utilizar para el seguimiento a largo plazo?.

El tratamiento definitivo es la intervención quirúrgica. La corrección quirúrgica implica la ligadura del vaso anómalo o el establecimiento de una perfusión coronaria dual9 ${ }^{9}$.

En nuestro caso, por la avanzada edad de la paciente, así como un adecuado control sintomático con terapia médica óptima, se desestimó el tratamiento quirúrgico. La paciente ha permanecido con angina de moderados esfuerzos y no ha requerido ingreso hospitalario.

\section{CONCLUSIÓN}

El síndrome de ALCAPA es una anomalía congénita rara en la que el tronco coronario izquierdo se origina a partir de la arteria pulmonar principal. Hay que considerar que, a pesar de ser una anomalía de origen congénito, no es exclusiva de las edades pediátricas, por lo que es importante tener en cuenta esta condición potencialmente mortal en los adultos ${ }^{4}$.

El diagnóstico se puede establecer mediante una angiografía por TC y/o RM cardíaca ${ }^{6-8}$. El tratamiento inmediato puede prevenir complicaciones potencialmente mortales, como el infarto de miocardio o la muerte súbita9.

\section{AGRADECIMIENTOS}

Los autores agradecen al Servicio de Cardioimagen y al personal que participó en la elaboración de este reporte de caso.

\section{CONFLICTO DE INTERESES}

Los autores declaran que no existe conflicto de intereses.

\section{FINANCIAMIENTO}

Este trabajo no requirió de financiamiento. 


\section{RESPONSABILIDADES ÉTICAS}

Protección de personas y animales. Los autores declaran que para esta investigación no se han realizado experimentos en seres humanos ni en animales.

Confidencialidad de los datos. Los autores declaran que han seguido los protocolos de su centro de trabajo sobre la publicación de datos de pacientes.

\section{Derecho a la privacidad y consentimiento} informado. Los autores han obtenido el consentimiento informado de los pacientes y/o sujetos referidos en el artículo. Este documento obra en poder del autor de correspondencia.

\section{BIBLIOGRAFÍA}

1. Ugalde H, Rozas S, Sanhueza MI, Yubini M, García S. Síndrome de ALCAPA en adulto. Caso clínico. Rev Med Chile. 2017;145:121-5.

2. Keith JD. The anomalous origin of the left coronary artery from the pulmonary artery. Br Heart J. 1959;21(2):149-61.

3. Yau JM, Singh R, Halpern EJ, Fischman D. Anomalous origin of the left coronary artery from the pulmonary artery in adults: A comprehensive review of 151 adult cases and a new diagnosis in a 53-year-old woman. Clin Cardiol. 2011;34: 204-10.

4. Peña E, Nguyen ET, Merchant N, Dennie C. ALCAPA syndrome: Not just a pediatric disease. Radiographics. 2009;29(2):553-65.

5. Romero MJ, Farach R, Romero F, Pastor L. Síndrome de Bland-WhiteGarland en un varón anciano. Cardiocore. 2014;4:169-171.

6. Horisaki T, Yamashita T, Yokoyama H, Urasawa K, Kitabatake A. Threedimensional reconstruction of computed tomographic images of anomalous origin of the left main coronary artery from the pulmonary trunk in an adult. Am J Cardiol. 2003; 92:898-9.

7. Khanna A, Torigian DA, Ferrari VA, Bross RJ, Rosen MA. Anomalous origin of the left coronary artery from the pulmonary artery in adulthood on CT and MRI. Am J Roentgenol. 2005;185:326-9.

8. Takenaga M, Matsuda J, Miyamoto N, Ikushima I, Koiwaya Y. Magnetic resonance imaging of Bland-White-Garland syndrome-A case of anomalous origin of the left coronary artery form the pulmonary trunk in a 22-year-old woman. Jpn Circ J. 1998;62:219-21.

9. Dodge-Khatami A, Mavroudis C, Backer CL. Anomalous Origin of the Left Coronary Artery from the Pulmonary Artery: Collective Review of Surgical Therapy. Ann Thorac Surg. 2002;74:946-55. 